\title{
Nanoimprinted chiral plasmonic substrates with three-dimensional nanostructures
}

Mingliang Zhang, ${ }^{1,2,3}$ Victor Pacheco-Peña, ${ }^{1}$ Yao $Y u,{ }^{2}$ Wenxiang Chen, ${ }^{1}$ Nicholas J. Greybush, ${ }^{2}$ Aaron Stein, ${ }^{6}$ Nader Engheta, ${ }^{1,2,4,5}$ Christopher B. Murray, ${ }^{2,3,{ }^{*}}$ Cherie R. $\operatorname{Kagan}^{1,2,3, *}$

1. Department of Electrical and Systems Engineering, University of Pennsylvania, Philadelphia, PA, 19104, United States

2. Department of Materials Science and Engineering, University of Pennsylvania, Philadelphia, PA, 19104, United States

3. Department of Chemistry, University of Pennsylvania, Philadelphia, PA, 19104, United States

4. Department of Physics and Astronomy, University of Pennsylvania, Philadelphia, PA, 19104, United States

5. Department of Bioengineering, University of Pennsylvania, Philadelphia, PA, 19104, United States

6. Center for Functional Nanomaterials, Brookhaven National Laboratory, Upton, NY, 11973, United States

*e-mail: kagan@seas.upenn.edu; cbmurray@sas.upenn.edu; 


\begin{abstract}
We report a large-area fabrication method to prepare chiral substrates patterned with arrays of multilayer, three-dimensional nanostructures using a combination of nanoimprint lithography and glancing angle deposition. Several structures are successfully fabricated using this method, including L-shaped, twisted arc and tri-layer twisted Au nanorod structures, demonstrating its generality. As one typical example, arrays of L-shaped nanostructures, consisting of two layers of orthogonally oriented Au nanorods separated by a Ge dielectric layer in the thickness direction, exhibit giant optical chirality in the infrared region with an experimentally achieved $g$-factor as high as 0.38 . Electromagnetic simulations show that the optical chirality results from plasmon hybridization between the two orthogonal Au segments. To demonstrate scalability, a $1-\mathrm{cm}^{2}$ chiral substrate is fabricated with uniform chiral optical property. This method combines both high throughput and precise geometrical control, and is therefore promising for applications of chiral metamaterials.
\end{abstract}

\title{
Key words
}

Chiral substrate, plasmonics, nanoimprinting lithography, glancing angle deposition 
Chirality is an intrinsic property of many non-planar molecules in which their atoms may be configured in three-dimensions (3D) such that the molecules lack inversion symmetry, creating enantiomers. ${ }^{1}$ Due to their structural handedness, these molecules interact differently with left-handed circularly polarized (LCP) and right-handed circularly polarized (RCP) light, leading to both intensity and phase difference in their transmitted waves. Circular dichroism (CD) is a chiral phenomenon characterizing the intensity difference in the transmittance spectra. The chiral response of natural molecular materials is weak, only detectable in thick samples when the incident light traverses a macroscopic path. In contrast, metamaterials consisting of artificially-engineered, sub-wavelength $3 \mathrm{D}$ chiral plasmonic nanostructures give rise to analogous structural handedness, yet giant chiral responses in ultrathin structures. ${ }^{2-13}$ The strong chiral response of metamaterials has opened up practical applications, including chemical and biological sensing 14-16 distinguishing the chirality in different biomolecules, and light manipulation such as circular polarizers. ${ }^{17,18}$

Unfortunately it is not trivial to fabricate low-cost, large-area 3D chiral substrates. Glancing-angle deposition (GLAD) is a low-cost, large-area technique used to create 3D chiral structures by rotating the substrate while depositing material at an oblique angle, ${ }^{2-5}$ but it is limited in the range of achievable geometries. Nanoimprint lithography (NIL) is also a low-cost patterning technique, which in contrast offers adequate flexibility in the geometrical design, but of planar structures ${ }^{19-22}$ with very few examples of $3 D$ configurations $^{23,24}$ as it lacks capabilities for nanoscale alignment. 3D multilayer chiral configurations are usually fabricated by non-scalable electron-beam lithography (EBL) and physical vapor deposition $6-10,12,15,17$ or focused ion beam-induced deposition (FIBID). ${ }^{13}$ 3D nanostructures show strong chiral responses unlike the weak, extrinsic chiral responses that have been reported in $2 \mathrm{D}$ metasurfaces,${ }^{25}$ whose chirality originates from 
differences in the dielectric environment between the top air and bottom substrate interfaces and/or the direction of illumination.

Here, we report the fabrication of large-area substrates composed of arrays of multilayer 3D chiral nanostructures using a combination of NIL and GLAD. Unlike conventional EBL processes, which require a number of alignment, patterning and deposition cycles, here we greatly simplify the fabrication. We use one nanoimprint patterning step and a multistep GLAD process in which, by changing the deposition direction, we sequentially deposit and stack different, well-defined planar nanostructures to create 3D chiral configurations. These multilayer chiral nanostructures show giant chiral responses in the infrared region, which is well explained by electromagnetic (EM) simulations. Using this method, a centimeter-sized chiral substrate has been fabricated with uniform chiral properties, further demonstrating the large-area fabrication of our combined NIL and GLAD method.

An array of L-shaped chiral plasmonic nanostructures is first demonstrated as an example. The fabrication process is illustrated in Figure 1A (detailed in Methods Section, Supporting Information). Here, a bilayer resist with a total thickness of $400 \mathrm{~nm}$ is patterned by NIL and developed ${ }^{26}$ to create arrays of L-shaped templates. An example L-shaped template has $\mathrm{x}$ - and $\mathrm{y}$ - segment lengths/widths of $1.1 \mu \mathrm{m} / 100 \mathrm{~nm}$ and $1.4 \mu \mathrm{m} / 130 \mathrm{~nm}$. The L-shaped template is repeated to form arrays with pitches in the $x$ - and $y$-directions of $1.5 \mu \mathrm{m}$ and 1.6 $\mu \mathrm{m}$. GLAD is first used to selectively deposit Au in the x-segment by controlling the deposition direction, such that its projection on the substrate is along the negative $x$-axis, and by maintaining a deposition angle of $48^{\circ}$ relative to the substrate normal. In this way, the bottom of the y-segment is completely blocked, but its side wall will be coated and consequently its width reduced. Therefore, a larger y-segment width of $130 \mathrm{~nm}$ is patterned in the template as compared with $100 \mathrm{~nm}$ x-segment width to account for the 
width reduction. Next, a second GLAD step is conducted with a deposition direction projected along the negative $y$-axis and the same deposition angle of $48^{\circ}$. This time, the x-segment is blocked and a stack of Ge and Au is sequentially deposited in the y-segment. After lift-off, arrays of L-shaped 3D nanostructures composed of two orthogonal layers of Au nanorods vertically separated by a Ge dielectric spacer layer are created.

Using different templates with $L$ and its inverse $\lrcorner$ shapes, we fabricate artificial pairs of enantiomers (Figure 1B, 1C, and Figure S1, Supporting Information). The first $\mathrm{Au} x$ segment has a length/width of $811 \pm 11 \mathrm{~nm} / 116 \pm 2 \mathrm{~nm}$ for Structure $L$ and $786 \pm 7 / 119 \pm 2 \mathrm{~nm}$ for Structure $\rfloor$. The second Ge/Au y-segment consists of two regions: a short tip region at the junction (highlighted in red circles) stacked on top of the first segment and a long flat region. The short tip region has a complex, humped structure due to the underlying $\mathrm{Au}$ segment, with a typical length of $180 \pm 17 \mathrm{~nm}$. Due to the angular deposition, there is also some Ge/Au material deposited beyond the short tip in the negative y direction. The long flat region has a length of $772 \pm 14 \mathrm{~nm}$ for Structure $L$ and $813 \pm 14 \mathrm{~nm}$ for Structure $\lrcorner$. By choosing the segment lengths in the template $(1.1 \mu \mathrm{m}$ in $\mathrm{x}$-segment and $1.4 \mu \mathrm{m}$ in $\mathrm{y}$ segment), the geometries of the deposited Au nanorods are optimized to have the largest chiral response (described below). In both regions, the widths are $108 \pm 2 \mathrm{~nm}$ and $92 \pm 2 \mathrm{~nm}$ for Structure $L$ and $\lrcorner$, respectively. The slight segment length difference between Structure $L$ and $\lrcorner$ is caused by the compromised angle alignment accuracy in the GLAD process using a simple, conventional thermal evaporator, and may be improved with a more advanced deposition setup with precise control of the deposition angle. The thicknesses are $29.2 \pm 1.0 \mathrm{~nm}$ for both Au layers in the $\mathrm{x}$ - and $\mathrm{y}$ - segment, and are $76.7 \pm 1.7 \mathrm{~nm}$ for the Ge layer (measured by atomic force microscopy, AFM, Figure S1, Supporting Information). Excluding the short tip region, we have realized a double-layer twisted Au nanorod array using a single NIL patterning step, which previously was only realized by a multilayer EBL 
patterning process. ${ }^{9,10}$ The combined NIL and GLAD process developed here allows us to fabricate uniform nanostructures over large areas (Figure 1d).

The circular polarization transmittance spectra of the enantiomeric $L$ and $\lrcorner$ chiral substrates are characterized by Fourier-transform Infrared (FTIR) spectroscopy (detailed in Methods Section, Supporting Information), and further compared with EM simulations in Figure 2. Two resonances (Figure $2 \mathrm{~A}$ and $2 \mathrm{~B}$ ) are found experimentally at 4710 $\mathrm{nm} / 5540 \mathrm{~nm}$ for Structure $\mathrm{L}$ and $4910 \mathrm{~nm} / 5450 \mathrm{~nm}$ for Structure $\lrcorner$, respectively, which also appear in the linear polarization transmittance spectra (Figure S3, Supporting Information). EM simulations confirm the presence of the two resonances (Figure $2 \mathrm{C}$ and 2D) at $4220 \mathrm{~nm} / 5630 \mathrm{~nm}$. Notably, the simulated NIL and GLAD-derived structures capture features in the y-segment, including a 65-nm Ge/20-nm Au sidewall connecting the short tip and long flat region and a $100 \mathrm{~nm}$ indent of the Au layer in the short tip region (Figure S2, Supporting Information). However, the simulated structure is still a simplified model, and does not take into account the smooth curvature in the short tip region, the trapezoidal cross section of the segments and the surface roughness, which are typical in experimentally deposited structures and can lead to both a shift and broadening of the peaks in experimental transmittance spectra. ${ }^{27}$ However, both experimental and simulated spectra have shown that Structure $L$ has a larger extinction for LCP-incident radiation at Resonance 1 (at shorter wavelength) and for RCP-incident radiation at Resonance 2 (at longer wavelength), and the situation is reversed for Structure $\lrcorner$. Further charge density analysis shows that the two resonances arise from different charge oscillation modes along the $x$ - and $y$ - Au nanorod segments. At any instance in time, the charge oscillations form a head-to-head dipolar anti-bonding configuration at Resonance 1 and a head-to-tail dipolar bonding configuration at Resonance 2 (Figure $2 \mathrm{G}$ and $2 \mathrm{H}$ ), as a result of plasmon 
hybridization. ${ }^{28}$ This is consistent with a number of studies on the interaction between two plasmonic nanorods..$^{29-32}$

The different response to circularly polarized light is a characteristic feature of enantiomers that arises from the different nature of the charge oscillations for the two resonance modes. Stronger coupling, and therefore larger extinction, occurs when the spatial electric field arrangement of the circularly polarized light matches the direction of the charge oscillation in the two Au segments. ${ }^{32}$ We use $\Delta T=T_{R C P} T_{L C P}$ to characterize the difference in the transmittances under RCP- and LCP- incident radiation, respectively. Due to geometrical symmetry, the $\Delta T$ spectra (Figure $2 E$ and $2 F$ ) for Structure $L$ and $\lrcorner$ have mirror-symmetry across the $\Delta T=0$ axis, and they show bisignate line shapes, consistent with the plasmonic Born-Kuhn model. ${ }^{10,32}$ The peak/dip positions in the $\Delta T$ spectra correlate with the two resonance positions in the transmittance spectra. The fabricated chiral substrates reach their $|\Delta \mathrm{T}|$ peak values of 0.077 (Structure $\mathrm{L}$ ) and 0.049 (Structure $\lrcorner$ ) at Resonance 2, while the simulated result has predicted an even higher value of 0.115 . The peak value difference between the enantiomers is attributed to the slight dimensional difference introduced in the fabrication process as detailed above, affecting their coupling efficiency to circularly polarized light. The $\Delta \mathrm{T}$ value of the nanoimprinted chiral substrate is comparable to that of reported EBL-patterned chiral nanostructures ${ }^{9}$ operating in a similar spectral range.

The flexibility of NIL template design has allowed us to adjust the segment lengths to further understand the optical responses of these chiral nanostructures. In both experiment and simulation, the $x$-segment length is kept fixed (at $752 \pm 39 \mathrm{~nm}$ in experiment and at $810 \mathrm{~nm}$ in simulations as obtained from Structure $L$ in Figure 1B), while the $y$ segment length (referring to the length of the long flat region, $L_{y}$ in Supporting Information, 
Figure S2) is varied from $300 \mathrm{~nm}$ to $900 \mathrm{~nm}$. Figure $3 \mathrm{~A}$ and Supporting Information Figure S4 show the evolution of the two resonance positions as the $y$-segment length is changed (Figure S5, Supporting Information). While both resonances red-shift with the increase of the $y$-segment length, Resonance 1 changes much more rapidly. At short y-segment lengths, the two resonances are well separated and the hybridization effect is negligible. Resonance 1 and 2 are dominated by the excitation of $y-(x-)$ polarized incident light (Figure S6, Supporting Information). As the y-segment length increases, the Resonance 1 wavelength increases and approaches that of Resonance 2, and the plasmon hybridization becomes strong. The $|\Delta T|$ peak amplitude reaches its maximum at a ysegment length of $700 \mathrm{~nm}$ (Figure 3B and Figure S7, Supporting Information), where the plasmon in the two Au segments are strongly hybridized. This is understandable as the effective coupling with circularly polarized light requires large electron oscillation in both Au segments to best match the rotating electric field. The experimentally measured $|\Delta T|$ peak amplitudes are consistent with this trend. As described earlier, the simulation does not take into account the smooth curvature in the short tip region, the trapezoidal cross section of the segments and the surface roughness, which are typical in experimentally deposited structures and will lead to both a shift and broadening of the peaks in experimental transmittance spectra. In fact, when the dissymmetry factor (g-factor) is calculated, ${ }^{4}$ which normalizes the difference in the extinction between RCP and LCP illumination to the unpolarized extinction, we find a better match between the experimental and simulated results (Figure $3 C$ ). This indicates that $|\Delta T|$ peak amplitudes can be further increased by patterning a higher density of the same nanostructures. The introduction of $g$-factor, in addition to $\Delta T$, serves to provide a figure-of-merit that can be used to fairly compare the intrinsic circular dichroism of different structures independent of their densities. Experimentally, we have reached the highest $g$-factor of 0.38 for the structure in Figure 2A at Resonance 2, which exceeds previously reported values for larger-area 
chiral substrates using high-throughput techniques. ${ }^{4,5,33}$ The improved performance is attributed to the better-defined structure realized using NIL. We notice that Gansel and coworkers ${ }^{18}$ have reached higher $g$-factors in the same wavelength range. However, their chiral plasmonic structures are patterned using direct laser writing, which is a serial patterning method, limiting the fabrication to small sample size.

The combined NIL and GLAD method can also be used to fabricate other multi-layer chiral structures. For example, earlier work has demonstrated the multilayer twisted arc structure as a promising candidate for generating a giant chiral response. ${ }^{10}$ To realize the largearea fabrication of this structure, we have designed an inverse-C-shaped template (Figure 4A and Figure S8A, Supporting Information), followed by a two-step deposition projected along the negative $\mathrm{x}$-axis $\left(32-\mathrm{nm} \mathrm{Au}\right.$, deposition angle $\left.43^{\circ}\right)$ and negative $\mathrm{y}$-axis $(77-\mathrm{nm}$ $\mathrm{Ge} / 29-\mathrm{nm} \mathrm{Au}$, deposition angle $48^{\circ}$ ), sequentially. The resultant structure shows two crescent-shaped plasmonic arcs separated by $\mathrm{Ge}$ in the vertical direction (Figure 4B and 4C). Similar to the L-shaped structures, the twisted arc structure shows two overlapping dips in its circular polarization transmittance spectra, and its $\Delta T$ spectrum peaks at 5100 $\mathrm{nm}$ (Figure S8B and S8C, Supporting Information), with a maximal value of 0.021 and a g-factor of 0.26 . Further increasing the structural complexity, we have also fabricated a trilayer twisted nanorod metasurface using a tri-branch 'windmill' template (Figure 4D and S8D, Supporting information). A three-step $48^{\circ}$ angular deposition is used, and in each step, the projected deposition direction is along one of the branches and the other two are blocked due to the shadowing effect. $29 \mathrm{~nm} \mathrm{Au}, 38 \mathrm{~nm} \mathrm{Ge} / 29 \mathrm{~nm} \mathrm{Au}$ and $77 \mathrm{~nm} \mathrm{Ge/29}$ $\mathrm{nm}$ Au are sequentially deposited in each branch in a clockwise manner, and consequently, these Au nanorods form a twisted configuration (Figure 4E and 4F), which can function as a broadband circular polarizer. ${ }^{17}$ The above examples show the versatility of this method in fabricating different chiral plasmonic nanostructures. 
To demonstrate the scalability of the combined NIL and GLAD process, we have used a centimeter-sized NIL template to fabricate a chiral substrate with Structure $\lrcorner$ (Figure 5A). The large-area template is patterned by EBL, ${ }^{20}$ but it can also be fabricated using edge and microlens projection lithography. ${ }^{19,34}$ All four corners and the center of the large-area chiral substrate are characterized and the $\Delta \mathrm{T}$ spectra consistently show similar bisignate line-shapes (Figure 5B). The $|\Delta \mathrm{T}|$ peak position and amplitude are measured to be $5230 \pm 10 \mathrm{~nm}$ and $0.041 \pm 0.003$ (Figure $5 \mathrm{C}$ ), respectively. The large-area uniformity of the optical performance confirms the scalable nature of this method.

In conclusion, we have realized the large-area fabrication of multilayer chiral plasmonic substrates using a combination of NIL and GLAD, which has previously been realized only by non-scalable EBL process. ${ }^{10,15}$ Several structures are successfully fabricated using this method, including L-shaped, twisted arc and tri-layer twisted Au nanorod structures. Due to both their structural chirality and plasmonic enhancement effect, these chiral plasmonic structures show strong interaction with circularly polarized light. Experimentally for Lshaped structures, we have found a $|\Delta T|$ peak amplitude as high as 0.08 , comparable to those patterned with non-scalable EBL process. We have also scaled up the chiral substrate size to $1 \mathrm{~cm}^{2}$ and the sample shows high uniformity optically over the entire sample surface. It is possible to shift the wavelength range into the visible and increase the magnitude of the chiral response by fabricating nanoimprinting stamps with $\sim 100 \mathrm{~nm}$ structures and in higher density.

The $C D$ of the fabricated chiral substrates $[0.044$ at Resonance 2, defined as $\left.[\Delta T\rangle /\left(T_{R C P}+T_{L C P}\right)\right]$ is sufficient for biomolecular sensing applications. ${ }^{14}$ The implementation 
of an ultrathin circular polarizer ideally requires much higher $C D$. By increasing the density of our L-shaped structures, by 3.5-fold for an example pattern design (Figure S9, Supporting Information), simulation indicates that the $C D$ can reach 0.130 . The $C D$ values can be further increased if multiple layers of the chiral plasmonic substrates are stacking together. For example, a 7-layer stack will lead to a $C D$ of 0.725 , comparable to that of a small-area, circular polarizer fabricated using serial, direct laser writing. ${ }^{18}$ These chiral plasmonic nanostructures can be further embedded in thermally or chemically responsive media, ${ }^{9,35}$ allowing the active modulation of their optical chirality, or with photo-luminescent materials, resulting in chiral selective enhancement of photon emission. ${ }^{36}$ Such a low-cost, large-area fabrication technique promises to produce chiral substrates with well-defined multilayer structures that can be practically used for applications in biosensing and light manipulation. 


\section{Acknowledgement}

The authors are grateful for primary support of this work from the National Science Foundation (NSF) under Award No. CMMI-1562884 for chiral structure fabrication and characterization. Electromagnetic simulation and SEM imaging was performed in facilities supported by the NSF MRSEC program under award No. DMR-1720530. E-beam lithography to pattern the nanoimprint lithography master stamp was carried out at the Center for Functional Nanomaterials, Brookhaven National Laboratory, which is supported by the U.S. Department of Energy, Office of Basic Energy Sciences, under Contract No. DE-AC02-98CH10886. YY was supported by Penn's Department of Materials Science and Engineering Masters Scholars Award.

The authors declare no competing financial interest.

Supporting Information available: methods' additional AFM, SEM and optical characterization; structural information used for simulation; design pattern and optical characterization for twisted-arc and tri-layer twisted Au nanorod structure. 


\section{References}

1. Ma, W.; Xu, L.; de Moura, A. F.; Wu, X.; Kuang, H.; Xu, C.; Kotov, N. A. Chem. Rev. 2017, 117, 8041-8093.

2. Mark, A. G.; Gibbs, J. G.; Lee, T. C.; Fischer P. Nat. Mater. 2013, 12, 802-807.

3. Frank, B.; Yin, X.; Schäferling, M.; Zhao, J.; Hein, S. M.; Braun, P. V.; Giessen H. ACS Nano 2013, 7, 6321-6329.

4. He, Y.; Larsen, G. K.; Ingram, W.; Zhao, Y. Nano Lett. 2014, 14, 1976-1981.

5. Yeom, B.; Zhang, H.; Zhang, H.; Park, J. I.; Kim, K.; Govorov, A. O.; Kotov, N. A. Nano Lett. 2013, 13, 5277-5283.

6. Plum, E.; Fedotov, V. A.; Schwanecke, A. S.; Zheludev, N. I. Appl. Phys. Lett. 2007, $90,223113$.

7. Liu, N.; Liu, H.; Zhu, S.; Giessen, H. Nat. Photon. 2009, 3, 157-162.

8. Hentschel, M.; Schäferling, M.; Metzger, B.; Giessen, H. Nano Lett. 2013, 13, 600606.

9. Yin, X.; Schäferling, M.; Michel, A. K. U.; Tittl, A.; Wuttig, M.; Taubner, T.; Giessen, H. Nano Lett. 2015, 15, 4255-4260.

10. Cui, Y.; Kang, L.; Lan, S.; Rodrigues, S.; Cai, W. Nano Lett. 2014, 14, 1021-1025.

11. Dietrich, K.; Lehr, D.; helgert, C.; Tünnermann, A.; Kley, E. B. Adv. Mater. 2012, 24, OP321-OP325.

12. Decker, M.; Ruther, M.; Kriegler, C. E.; Zhou, J.; Soukoulis, C. M.; Linden, S.; Wegener, M. Opt. Lett. 2009, 34, 2501-2503.

13. Esposito, M.; Tasco, V.; Todisco, F.; Cuscunà, M.; Benedetti, A.; Sanvitto, D.; Passaseo, A. Nat. Commun. 2015, 6, 6484. 
14. Hendry, E.; Carpy, T.; Johnston, J.; Popland, M.; Mikhaylovskiy, R. V.; Lapthorn, A. J.; Kelly, S. M.; Barron, L. D.; Gadegaard, N.; Kadodwala, M. Nat. Nanotechnol. 2010, 5, 783-787.

15. Zhao, Y.; Askarpour, A. N.; Sun, L.; Shi, J.; Li, X.; Alù, A. Nat. Commun. 2017, 8, 14180.

16. McPeak, K. M.; van Engers, C. D.; Bianchi, S.; Rossinelli, A.; Poulikakos, L. V.; Bernard, L.; Herrmann, S.; Kim, D. K.; Burger, S.; Blome, M.; Jayanti, S. V.; Norris, D. J. Adv. Mater. 2015, 27, 6244-6250.

17. Zhao, Y.; Belkin, M. A.; Alù, A. Nat. Commun. 2012, 3, 870.

18. Gansel, J. K.; Thiel, M.; Rill, M. S.; Decker, M.; Bade, K.; Saile, V.; von Freymann, G.; Linden, S.; Wegener, M. Science 2009, 325, 1513-1515.

19. Zhang, M.; Bechstein, D. J. B.; Wilson, R. J.; Wang, S. X. Nano Lett. 2014, 14, 333-338.

20. Zhang, M.; Magagnosc, D. J.; Liberal, I.; Yu, Y.; Yun, H.; Yang, H.; Wu, Y.; Guo, J.; Chen, W.; Shin, Y. J.; Stein, A.; Kikkawa, J. M.; Engheta, N.; Gianola, D. S.; Murray, C. B.; Kagan, C. R. Nat. Nanotechnol. 2017, 12, 228-232.

21. Chen, W.;Tymchenko, M.; Gopalan, P.; Ye, X.; Wu, Y.; Zhang, M.; Murray, C. B.; Alù, A.; Kagan, C. R. Nano Lett. 2015, 15, 5254-5260.

22. Lucas, B. D.; Kim, J. S.; Chin, C.; Guo, L. J. Adv. Mater. 2008, 20, 1129-1134.

23. Wi, J. S.; Barnard, E. S.; Wilson, R. J.; Zhang, M.; Tang, M.; Brongersma, M. L.; Wang, S. X. ACS Nano 2011, 5, 6449-6457.

24. Wi, J. S.; Sengupta, S.; Wilson, R. J.; Zhang, M.; Tang, M.; Wang, S. X. Small 2011, 7, 3276-3280.

25. Kuwata-Gonokami, M.; Saito, N.; Ino, Y.; Kauranen, M.; Jefimovs, K.; Vallius, T.; Turunen, J.; Svirko, Y. Phys. Rev. Lett. 2005, 95, 227401. 
26. Zhang, M.; Large, N.; Koh, A. L.; Cao, Y.; Manjavacas, A.; Sinclair, R.; Nordlander, P.; Wang, S. X. ACS Nano 2015, 9, 9331-9339.

27. Caglayan, H.; Hong, S. H.; Edwards, B.; Kagan, C. R.; Engheta, N. Phys. Rev. Lett. 2013, 111, 073904.

28. Prodan, E.; Radloff, C.; Halas, N. J.; Nordlander, P. Science 2003, 302, 419-422.

29. Smith, K. W.; Zhao, H.; Zhang, H.; Sánchez-Iglesias, A.; Grzelczak, M.; Wang, Y.; Chang, W. S.; Nordlander, P.; Liz-Marzán, L. M.; Link, S. ACS Nano 2016, 10, 6180-6188.

30. Yang, S.; Ni, X.; Yin, X.; Kante, B.; Zhang, P.; Zhu, J.; Wang, Y.; Zhang, X. Nat. Nanotechnol. 2014, 9, 1002-1006.

31. Chu, M. W.; Myroshnychenko, V.; Chen, C. H.; Deng, J. P.; Mou, C. Y.; de Abajo, F. J. G. Nano Lett. 2009, 9, 399-404.

32. Yin, X.; Schäferling, M.; Metzger, B.; Giessen, H. Nano Lett. 2013, 13, 6238-6243.

33. Singh, J. H.; Nair, G.; Ghosh, A.; Ghosh, A. Nanoscale 2013, 5, 7224-7228.

34. Gonidec, M.; Hamedi, M. M.; Nemiroski, A.; Rubio, L. M.; Torres, C.; Whitesides, G. M. Nano Lett. 2016, 16, 4125-4132.

35. Duan, X.; Kamin, S.; Sterl, F.; Giessen, H.; Liu, N. Nano Lett. 2016, 16, 1462-1466. 36. Rodrigues, S. P.; Cui, Y.; Lan, S.; Kang, L.; Cai, W. Adv. Mater. 2015, 27, 11241130. 


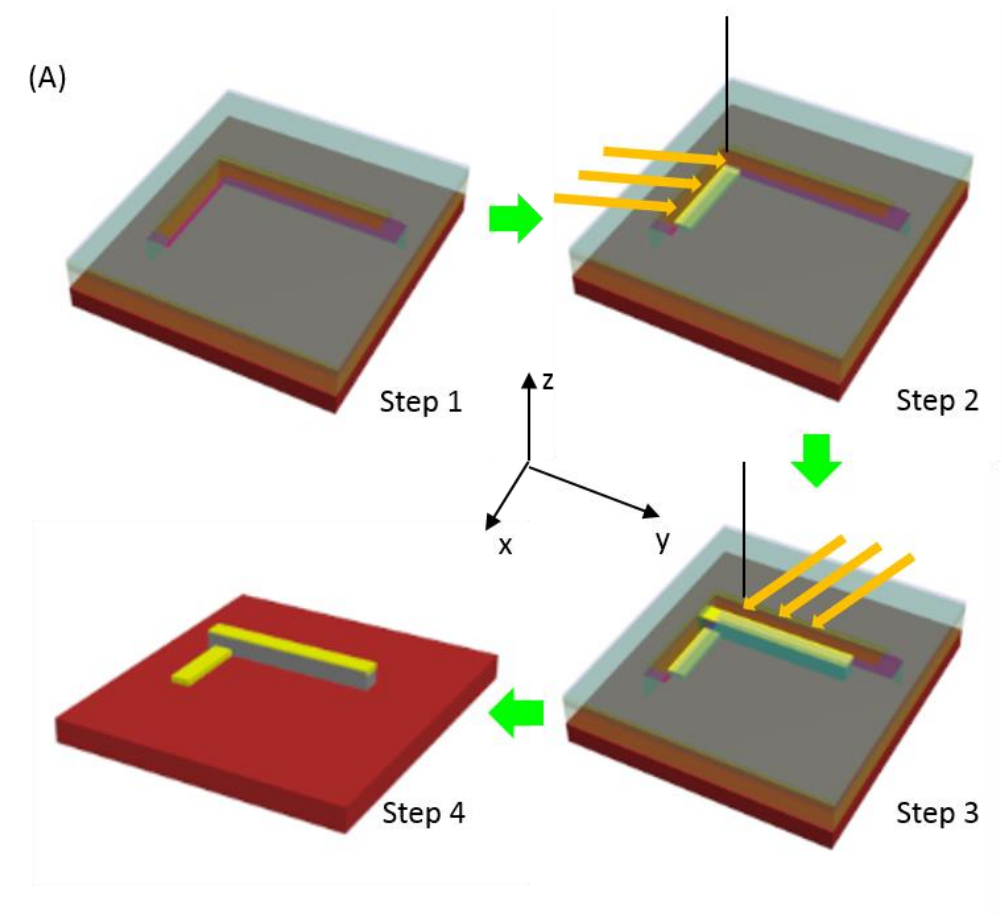

(B)

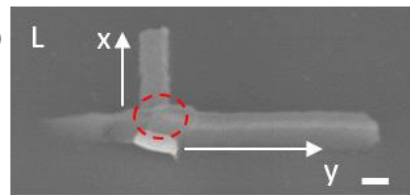

(C)

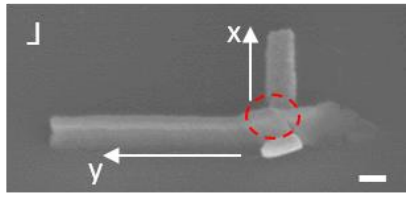

(D)

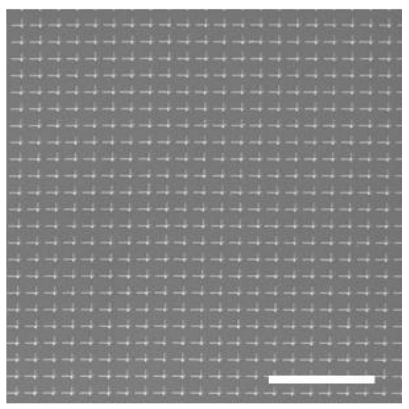

Figure 1. (A) Schematic of the fabrication process for L-shaped multilayer chiral nanostructures. Step 1: a bilayer resist is spincoated onto a double-side-polished Si wafer and patterned with L-shaped templates by NIL; Step 2: first GLAD process, depositing Au only in the x-segment; Step 3: second GLAD process, depositing Ge/Au only in the ysegment; Step 4: lift-off. (B, C) SEM images at a $45^{\circ}$ tilt angle for enantiomers of the chiral structure, $L$ and $\lrcorner$. Red circles highlight the short tip regions. (D) Low-magnification SEM image of an array of chiral nanostructures. Scale bars: (B, C) $100 \mathrm{~nm}$; (D) $10 \mu \mathrm{m}$. 


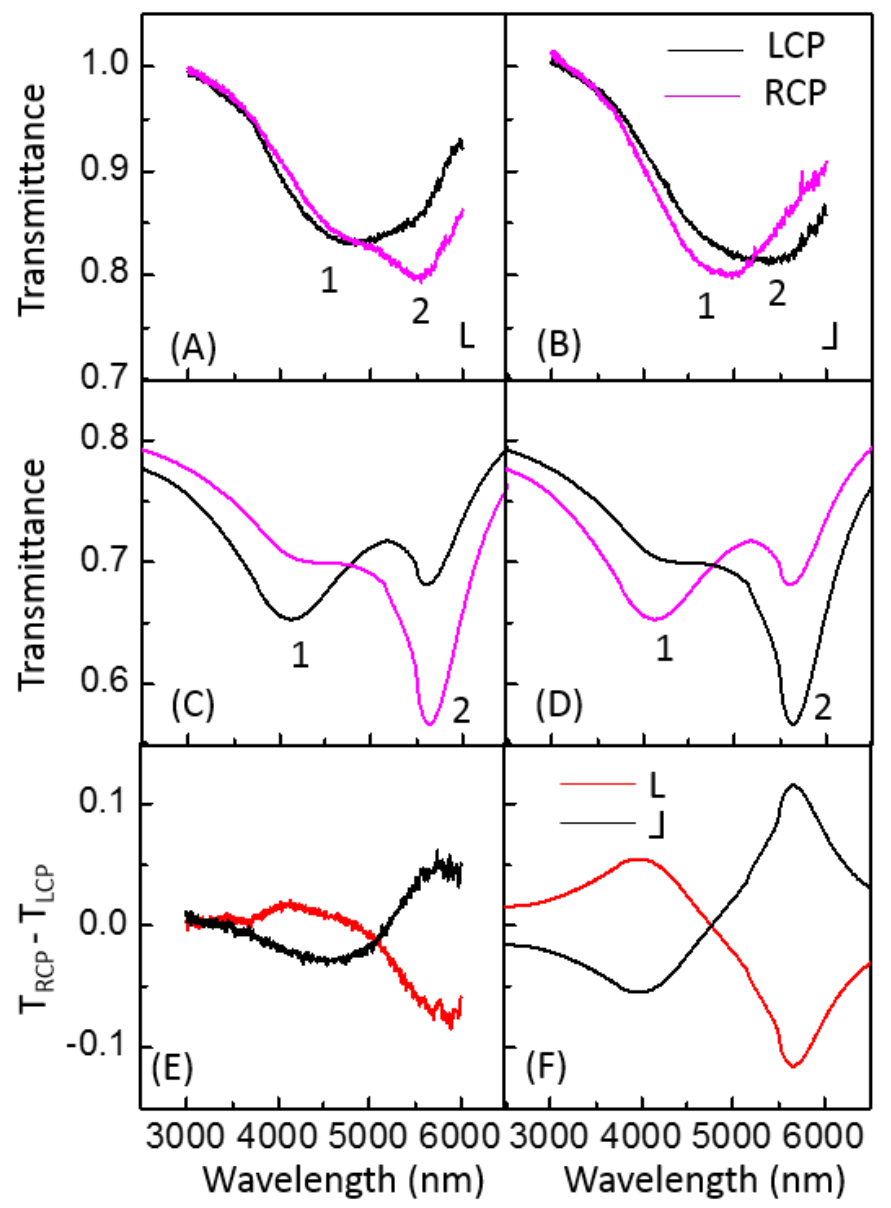

(G)

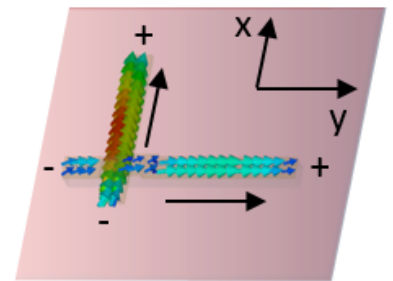

Resonance 1
$(\mathrm{H})$

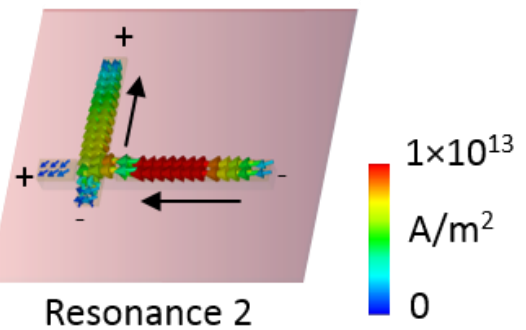

Figure 2. Circular polarization transmittance spectra in experiment for (A) Structure $L$ and (B) Structure $\lrcorner$ substrates, and in simulation for (C) Structure $L$ and (D) Structure $」$ substrates. (E) Experimental and $(F)$ simulated $\Delta T=T_{R C P}-T_{L C P}$ spectra for Structure $L$ and \lrcorner . Current density plots of Structure $L$ at $(G)$ Resonance 1 and $(H)$ Resonance 2. The plots correspond to LCP-incident light for Resonance 1 and RCP-incident light for Resonance 2, respectively. 


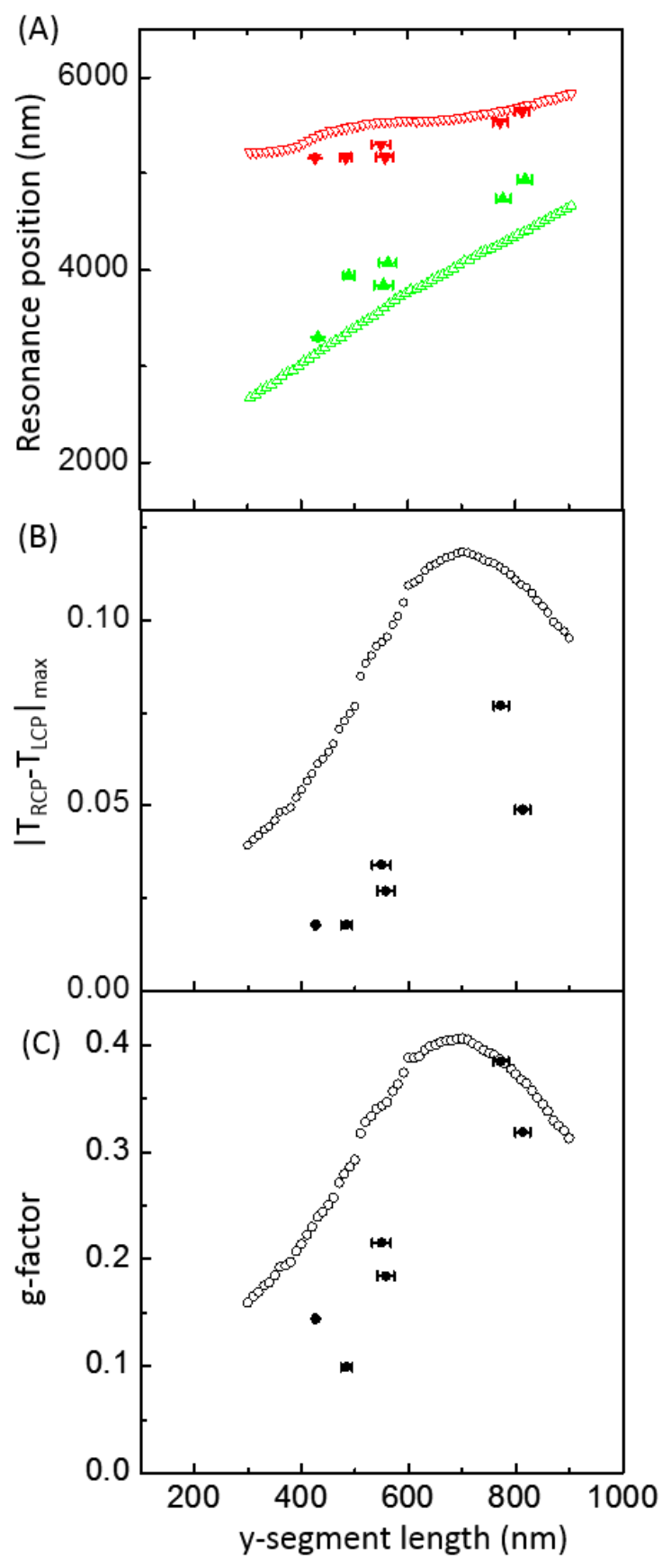

Figure 3. (A) Position of Resonance 1 (green triangles) and Resonance 2 (inverted red triangles) of the chiral nanostructures as a function of $y$-segment length. (B) $|\Delta T|$ peak amplitudes and (C) corresponding g-factors (at Resonance 2) as a function of the $y$ segment length. For all plots, the solid symbols are from experiment and the open symbols from simulations. 
(A)

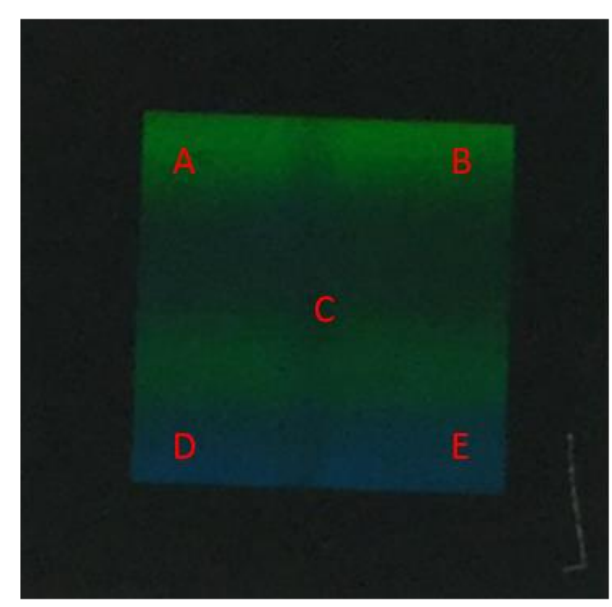

(C)

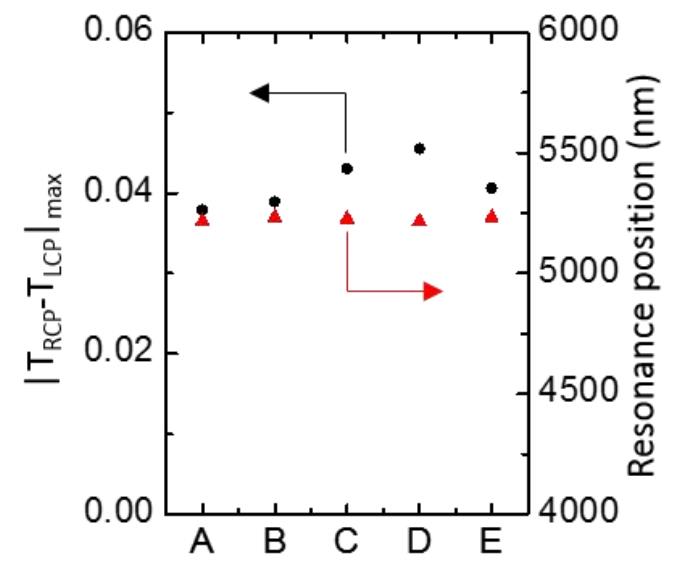

(B)

(B) 0.06

Spot $A$

0.03

0.00

-0.03
-0.06

0.06

Spot B

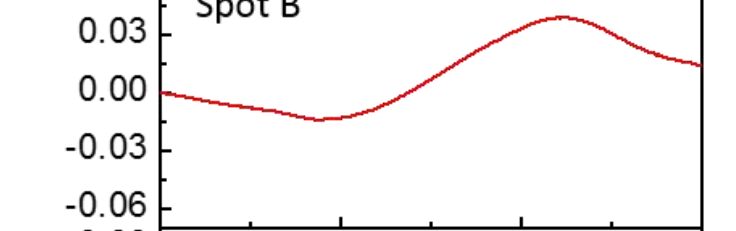

苂 0.0

Spot C

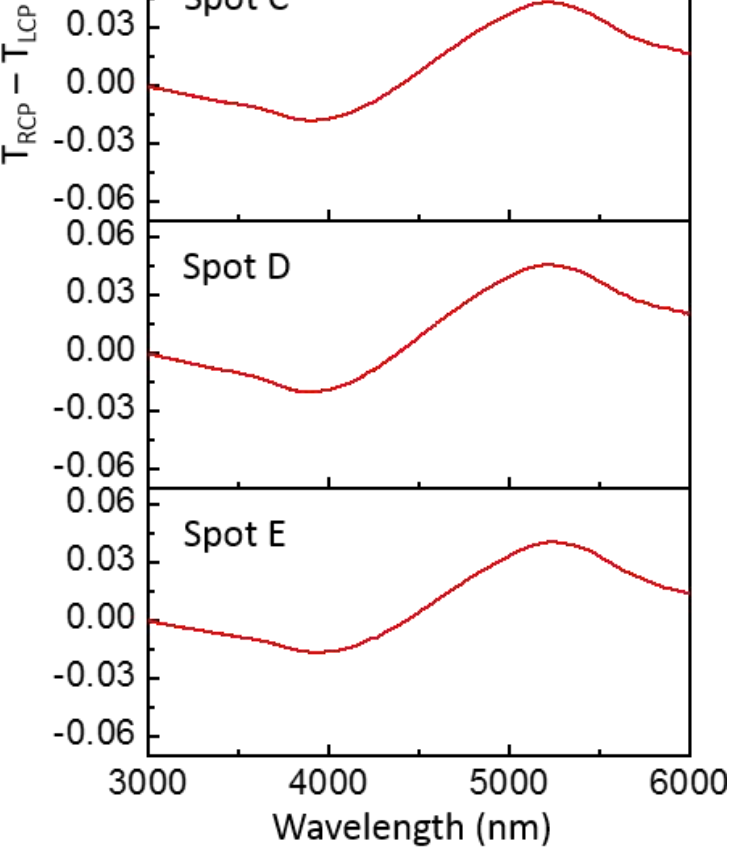

Figure 4. (A) Photograph of a $1-\mathrm{cm}^{2}$ chiral plasmonic substrate patterned with an array of Structure $\lrcorner$. Spots A, B, C, D and E are sampled to characterize the optical properties of the substrate. (B) $\Delta \mathrm{T}$ spectra and $(\mathrm{C})|\Delta \mathrm{T}|$ peak amplitudes (black dots) and positions (red triangles) at different sampling points. 